\title{
Different Roles for Orbitofrontal Cortex and Basolateral Amygdala in a Reinforcer Devaluation Task
}

\author{
Charles L. Pickens, Michael P. Saddoris, Barry Setlow, Michela Gallagher, Peter C. Holland, and Geoffrey Schoenbaum \\ Department of Psychological and Brain Sciences, Johns Hopkins University, Baltimore, Maryland 21218
}

The orbitofrontal cortex (OFC) and basolateral amygdala (BLA) are critical for using learned representations of outcomes to guide behavior. Neurophysiological findings suggest complementary roles in which the BLA acquires associations between cues and outcomes and the OFC subsequently uses them to guide behavior. Here, we have used a reinforcer devaluation paradigm to test this hypothesis. In this paradigm, rats are first trained to associate a light conditioned stimulus (CS) with a food outcome, and then the food is devalued by pairing it with illness. After this devaluation procedure, responding to the CS is assessed in a single probe session. Previously, we have shown that BLA and OFC lesions made before training do not affect the acquisition of conditioned responding but do impair the sensitivity of that responding to reinforcer devaluation. Rats with such lesions fail to exhibit the spontaneous decrease in conditioned responding to the light cue observed in controls in the probe test. Here, we have extended those findings by showing that performance in the probe test is impaired by OFC lesions made after light-food conditioning but not by BLA lesions made after that training. These findings indicate that the OFC and BLA play different roles in mediating normal goal-directed performance in this, and likely other, settings. The BLA seems critical to forming representations linking cues to the incentive properties of outcomes but not for maintaining these representations in memory, updating them with new information, or for expressing them in behavior. In contrast, the OFC seems essential for one or more of these latter processes.

Key words: basolateral amygdala; orbitofrontal cortex; devaluation; incentive value; Pavlovian conditioning; learning

\section{Introduction}

Anticipation of the emotional or motivational consequences of current events or actions serves an important adaptive function. Clinical evidence suggests that the ability to use learned expectations of such consequences to guide behavior is impaired in patients with damage to the ventromedial prefrontal cortex (vmPFC) or the amygdala. For example, performance is impaired in patients with damage to either of these brain regions in a gambling task that requires participants to guide their actions through experience with the relative payoffs and penalties associated with their choices (Bechara et al., 1999).

Related experiments have implicated the strong anatomical interconnections between the basolateral region of the amygdala (BLA) and orbitofrontal cortex (OFC) in animal models of goalguided action using reinforcer devaluation tasks (Hatfield et al., 1996; Gallagher et al., 1999). Normal performance of rats in these tasks (Holland and Straub, 1979) depends on the ability of a conditioned stimulus (CS) to gain access to the motivational sig-

Received Aug. 26, 2003; revised 0ct. 7, 2003; accepted 0ct. 10, 2003.

This work was supported by National Institute of Mental Health Grant MH60179 (M.G.), Mentored Clinical Scientist Award K08-AG00882 (G.S.), and National Science Foundation Graduate Research Fellowship DGE-0202743 (C.L.P.). Experiment 2 appeared in abstract form at the 32nd Annual Meeting of the Society for Neuroscience, November 2002. We thank Helena Winfield, Stephanie DiGuiseppi, Summer Nugent, Erin Kerfoot, and Vanessa McKenna for excellent technical support.

Correspondence should be addressed to Dr. Peter C. Holland, 3400 North Charles Street, 222 Ames Hall, Baltimore MD 21218. E-mail: pch@jhu.edu.

G. Schoenbaum's present address: Department of Anatomy and Neurobiology, University of Maryland School of Medicine, 685 West Baltimore Street, Health Sciences Facility-1, Room 280K, Baltimore, MD 21201.

Copyright $\odot 2003$ Society for Neuroscience $\quad$ 0270-6474/03/2311078-07\$15.00/0 nificance of an impending reinforcer. To test this function, rats are first trained to associate a light CS with a food reinforcer. Next, the incentive value of the food is reduced by pairing it with a toxin in the home cage, in the absence of the light. When the light is subsequently presented by itself in testing, conditioned responding to it is spontaneously reduced, appropriate to the new value of the food. Rats with lesions of either the BLA or OFC show normal acquisition of conditioned responding to the light and normal reduction of their consumption of the food after foodtoxin pairings but do not spontaneously decrease their responses in subsequent testing with the light alone (Hatfield et al., 1996; Gallagher et al., 1999). Similar results have been reported in an instrumental learning analog of this task in rhesus monkeys. Monkeys with lesions of the BLA (Malkova et al., 1997) or with lesions that functionally disconnect the BLA and OFC (Baxter et al., 2000) failed to reduce conditioned responding appropriately after devaluation of a food reinforcer by selective satiation.

In the studies just described, lesions were made before all training. Thus, deficits may reflect impairment in the acquisition of the ability of the CS to access the incentive value of the reinforcer in initial training, the updating of that value in aversion learning/satiation, or the use of an updated representation of that value in guiding final performance. To clarify the roles of the OFC and BLA in the devaluation task, we tested rats with lesions of the OFC (experiment 1) or the BLA (experiment 2) made after the completion of light-food training. We were guided by a model (Schoenbaum et al., 1999, 2000) in which the BLA is primarily involved in the initial acquisition of access of a CS to a represen- 
Table 1. Outline of experimental procedures

\begin{tabular}{lllll}
\hline Group & Light conditioning & Surgery & Taste aversion training & Devaluation probe test \\
\hline Paired & House light $\rightarrow$ food & Neurotoxic or sham lesions & Food $\rightarrow$ LiCl & House light \\
Unpaired & House light $\rightarrow$ food & Neurotoxic or sham lesions & Food, LiCl & Food \\
\hline
\end{tabular}

Light conditioning, devaluation probe test, and the second taste aversion test were conducted in the experimental chambers, whereas taste aversion training and the first taste aversion test were conducted in the home cages. $\rightarrow$, Paired presentations; Food, LiCl, unpaired presentations.

tation of reinforcer properties, including its incentive value, whereas the OFC is critical to response generation, selection, or decision processes (Jones and Mishkin, 1972). Within this model, lesions of the BLA made after the light has already gained access to a food representation should have no effect on devaluation test performance, whereas OFC damage should impair devaluation performance, regardless of when the lesions are made.

\section{Materials and Methods}

Subjects. Male Long-Evans rats (Charles River Laboratories, Raleigh, NC), which weighed 300-325 gm on arrival to the vivarium, were housed individually with ad libitum access to food and water, except during testing. The vivarium was climate controlled and illuminated from 6:00 A.M. to 8:00 P.M. For testing, the rats were food deprived to $85 \%$ of their baseline weights by limiting their access to food to a single daily meal. Food deprivation began $\sim 5 \mathrm{~d}$ before the start of testing and continued throughout training.

Surgical procedures. Aseptic surgeries to make bilateral neurotoxic lesions of the OFC (experiment 1) or BLA (experiment 2) were performed under Nembutal (50 mg/kg; Abbott Laboratories, Chicago, IL) or isoflurane (Isovet; Mallinckrodt, Mundelein, IL) anesthesia. All surgeries were conducted as shown in Table 1, after light conditioning and before taste aversion training. Neurotoxic lesions were made using NMDA at a concentration of $20 \mathrm{mg} / \mathrm{ml}$ in PBS (Sigma, St. Louis, MO). NMDA or vehicle was infused at a rate of $0.1 \mu \mathrm{l} / \mathrm{min}$.

For experiment 1, OFC lesions were made in 22 rats, using four injection sites in each hemisphere: two injections at $4.0 \mathrm{~mm}$ anterior to bregma, $4.2 \mathrm{~mm}$ ventral from the skull surface at bregma, and placed 2.2 $\mathrm{mm}(0.08 \mu \mathrm{l})$ and $3.7 \mathrm{~mm}(0.08 \mu \mathrm{l})$ from the midline; and two injections at $3.0 \mathrm{~mm}$ anterior to bregma, $5.2 \mathrm{~mm}$ ventral from the skull surface at bregma, and placed $4.2 \mathrm{~mm}(0.08 \mu \mathrm{l})$ and at $3.2 \mathrm{~mm}(0.05 \mu \mathrm{l})$ from midline. All injections were made using a glass micropipette attached by a length of plastic tubing to a picospritzer (General Valve Corporation, Fairfield, NJ). For 16 control rats, the glass micropipette was lowered to the same sites, but no injections were given.

For experiment 2, BLA lesions were made in 42 rats, using two injection sites in each hemisphere. Injections were made $2.8 \mathrm{~mm}$ posterior to bregma and $5.0 \mathrm{~mm}$ from the midline, at $8.4 \mathrm{~mm}(0.15 \mu \mathrm{l})$ and $8.7 \mathrm{~mm}$ $(0.25 \mu \mathrm{l})$ ventral from the skull surface at bregma. Injections of the PBS vehicle alone were made in 19 sham control rats at these same sites. These microinjections were made using a $1.0 \mu \mathrm{l}$ Hamilton syringe. One rat was unable to be anesthetized and served as a nonsurgical control.

Apparatus. The behavioral training apparatus consisted of eight individual chambers $(22.9 \times 20.3 \times 20.3 \mathrm{~cm})$ with aluminum front and back walls, clear acrylic sides and top, and a floor made of $0.48 \mathrm{~cm}$ stainless steel rods spaced $1.9 \mathrm{~cm}$ apart. A dimly illuminated food cup was recessed in the center of one end wall. An infrared photocell placed just inside the food cup was polled $(1 \mathrm{kHz})$ by computer circuitry. Each chamber was enclosed in a sound-resistant shell. A $6 \mathrm{~W}$ house light was mounted on the inside wall of the shell, $10 \mathrm{~cm}$ above the experimental chamber and even with the end wall opposite the food cup. Ventilation fans provided masking noise $(70 \mathrm{~dB})$. Constant dim illumination was provided by a $6 \mathrm{~W}$ lamp behind a dense red lens mounted on the ceiling of the shell. A television camera was mounted within each shell to provide a view of the chamber; the output from each camera was digitized, merged into one of two composite images of four of the chambers, displayed on a monitor, and recorded on videotape. Behavioral data from the videotapes are not reported here.

Behavioral training procedures. Table 1 shows an outline of the training procedures that were used in both experiment 1 and experiment 2 . Once the rats had been food deprived to $85 \%$ of their baseline body weight, they were trained to eat from the recessed food cup in a single $64 \mathrm{~min}$ shaping session that included 16 deliveries of the reinforcer, two $45 \mathrm{mg}$ Noyes food pellets (P. J. Noyes, Manchester, NH). Next, the rats received light conditioning (Table 1) in eight daily 64 min sessions. Each session consisted of $1610 \mathrm{sec}$ presentations of the $6 \mathrm{~W}$ house light CS, followed immediately by the delivery of two $45 \mathrm{mg}$ food pellet reinforcers. After light conditioning, the rats underwent surgery, followed by $12-15 \mathrm{~d}$ of recovery.

After recovery from surgery, the rats in each lesion condition were assigned to paired and unpaired groups for taste aversion training (Table 1). This training was designed to give both groups of rats the same exposure to the food pellets and lithium chloride ( $\mathrm{LiCl}$ )-induced illness but permit only the rats in the paired group to experience illness in conjunction with the food. Taste aversion training took place in the rats' home cages over $4 \mathrm{~d}$. On the first and third days, the rats in the paired group received $10 \mathrm{~min}$ access to a ceramic bowl containing 100 food pellets, identical to those delivered in the experimental chambers. Immediately after this 10 min period, the ceramic bowl was removed, and rats in both the paired and unpaired groups received an injection of $0.3 \mathrm{M} \mathrm{LiCl}$ solution $(5 \mathrm{ml} / \mathrm{kg}$, i.p.). On the second and fourth days of aversion training, the rats in the unpaired group received 10 min access to the ceramic bowls containing 100 food pellets in the experimental chambers, but no injections were given. (In experiment 2, six rats that consumed $<20$ pellets on the initial food pellet presentation were given an additional 10 min exposure to the pellets on the second exposure, to ensure that all paired rats had sufficient experience with the food to form a taste aversion.)

After taste aversion training, the rats received the devaluation probe test (Table 1). This $64 \mathrm{~min}$ session consisted of 16 presentations of the 10 sec house light, but no food pellets were delivered. Six hours later, the rats received $10 \mathrm{~min}$ access to $5045 \mathrm{mg}$ food pellets (those used as the reinforcer) placed in the food cup of the experimental chamber, to assess the level of generalization of the taste aversion from the home cage to the experimental chamber (aversion test) (Table 1). Finally, on the next day of testing, the rats received, in their home cages, 10 min access to a ceramic bowl containing 100 food pellets, as in the taste aversion training (aversion test) (Table 1).

Response measures. The primary measure of appetitive conditioning to the house light CS was the percentage of time the rat spent with its head in the food cup during the last $5 \mathrm{sec}$ of the $10 \mathrm{sec}$ CS and during the $5 \mathrm{sec}$ empty interval immediately before each CS, as indicated by disruption of the photocell beam. Previous data (Holland, 1977) show that food cup behaviors during $10 \mathrm{sec}$ visual CS are concentrated in the last $5 \mathrm{sec}$ of the cue. The consumption of food pellets in the home cage was determined by counting the number of pellets in the bowl after $10 \mathrm{~min}$; consumption in the experimental chamber test was determined by counting the number of pellets in the food cup and tray beneath the floor after $10 \mathrm{~min}$.

Histological procedures. After completion of behavioral testing, the rats were deeply anesthetized with Nembutal $(150 \mathrm{mg} / \mathrm{kg})$ and perfused with $0.1 \mathrm{~m}$ PBS, followed by $10 \%(\mathrm{v} / \mathrm{v})$ formalin. The brains were removed and stored in $0.1 \mathrm{M}$ PBS with $20 \%(\mathrm{w} / \mathrm{v})$ sucrose and $1 \%(\mathrm{w} / \mathrm{v})$ DMSO at $4^{\circ} \mathrm{C}$ for $24-48 \mathrm{hr}$. Sections $(60 \mu \mathrm{m})$ were taken from each brain through the OFC and BLA and mounted on slides and Nissl-stained to verify lesions.

\section{Results}

\section{Experiment 1: effect of postconditioning OFC lesions}

OFC lesion histology

One of the 16 sham control rats died soon after surgery; data from the remaining sham control rats (eight paired and seven un- 
paired) were included in the analysis. Of the 22 OFC-lesioned rats, one rat was excluded for failing to acquire conditioning before surgery. Nineteen ( 9 paired and 10 unpaired) of the remaining rats had acceptable lesions of the OFC, encompassing medial, ventrolateral, and lateral orbital regions and both dorsal and ventral agranular insular cortex. This target region was designed to include areas on the dorsal bank of the rhinal sulcus that have reciprocal interactions with the BLA (Krettek and Price, 1977; Kita and Kitai, 1990; Shi and Cassell, 1998), without extending into gustatory regions located in agranular insular cortex posterior to the genu of the corpus callosum (Saper, 1982; Kosar et al., 1986a,b; Krushel and Van Der Kooy, 1988). Lesions averaged $76 \%$ damage of this region bilaterally, ranging from 65 to $90 \%$. Lesioned rats were excluded $(n=2)$ if damage to the OFC was $<50 \%$ in either hemisphere or if there was extensive bilateral damage to cortical areas outside the target region, particularly gustatory cortex. There was no discernable damage in any of the sham-lesioned control rats. Examples of a representative lesion and sham brain are seen in photomicrographs in Figure 1, $a$ and $b$.

\section{Presurgical performance (light-food conditioning)}

All groups increased their food cup responses during the light over the course of training. Performance on the final conditioning session (Table 2) did not differ as a function of later lesion status or assignment to paired or unpaired taste aversion training. Although pre-CS response levels (Table 2) seemed lower in the rats that were assigned to the sham-paired condition than the sham-unpaired condition, this difference was not statistically significant. Separate lesion $\times$ taste aversion ANOVAs of responding during both the CS and pre-CS periods found no significant main effects or interactions (all $p>0.05$ ).

\section{Postsurgical performance (taste aversion and devaluation} probe test)

Taste aversion training caused an equivalent reduction of consumption in both paired groups over the course of that training, whereas neither of the unpaired groups showed a reduction in consumption (Fig. $2 A$ ). A lesion $\times$ taste aversion $\times$ trial ANOVA found a significant effect of taste aversion $\left(F_{(1,30)}=32.05 ; p<\right.$ $0.001)$, trial $\left(F_{(2,60)}=32.90 ; p<0.001\right)$, and a significant interaction of taste aversion $\times \operatorname{trial}\left(F_{(2,60)}=81.96 ; p<0.001\right)$. No other main effects or interactions were significant (all $p>0.05$ ). Furthermore, the taste aversion transferred readily from the home cage in which it was established to the experimental chamber, and that transfer was unaffected by the OFC lesions. A lesion $\times$ taste aversion ANOVA of the data from the experimental chamber consumption test (Fig. $2 \mathrm{~A}$, rightmost points) found a significant effect of taste aversion $\left(F_{(1,30)}=598.56 ; p<0.001\right)$. No other main effects or interactions were significant (all $p>$ $0.05)$.

Figure $2 B$ shows the results of the probe test session, the primary data of interest. For consistency with our data analyses in previous studies of devaluation from this laboratory, we present the results of the first eight test trials only. There was a significant devaluation effect in the sham rats but not in the OFC-lesioned rats. Sham-paired rats responded less during the light CS than sham-unpaired rats $\left(F_{(1,30)}=4.19 ; p<0.05\right)$, but responding of OFC-paired and OFC- unpaired rats did not differ $(F<1)$. Responding was also significantly greater in the OFC-paired rats than in the sham-paired rats $\left(F_{(1,30)}=4.95 ; p<0.05\right)$, consistent with a diminished influence of changes in the value of the food pellets in the lesioned rats.

A similar behavioral pattern was also observed during the pre-CS periods. Pre-CS responding was significantly lower
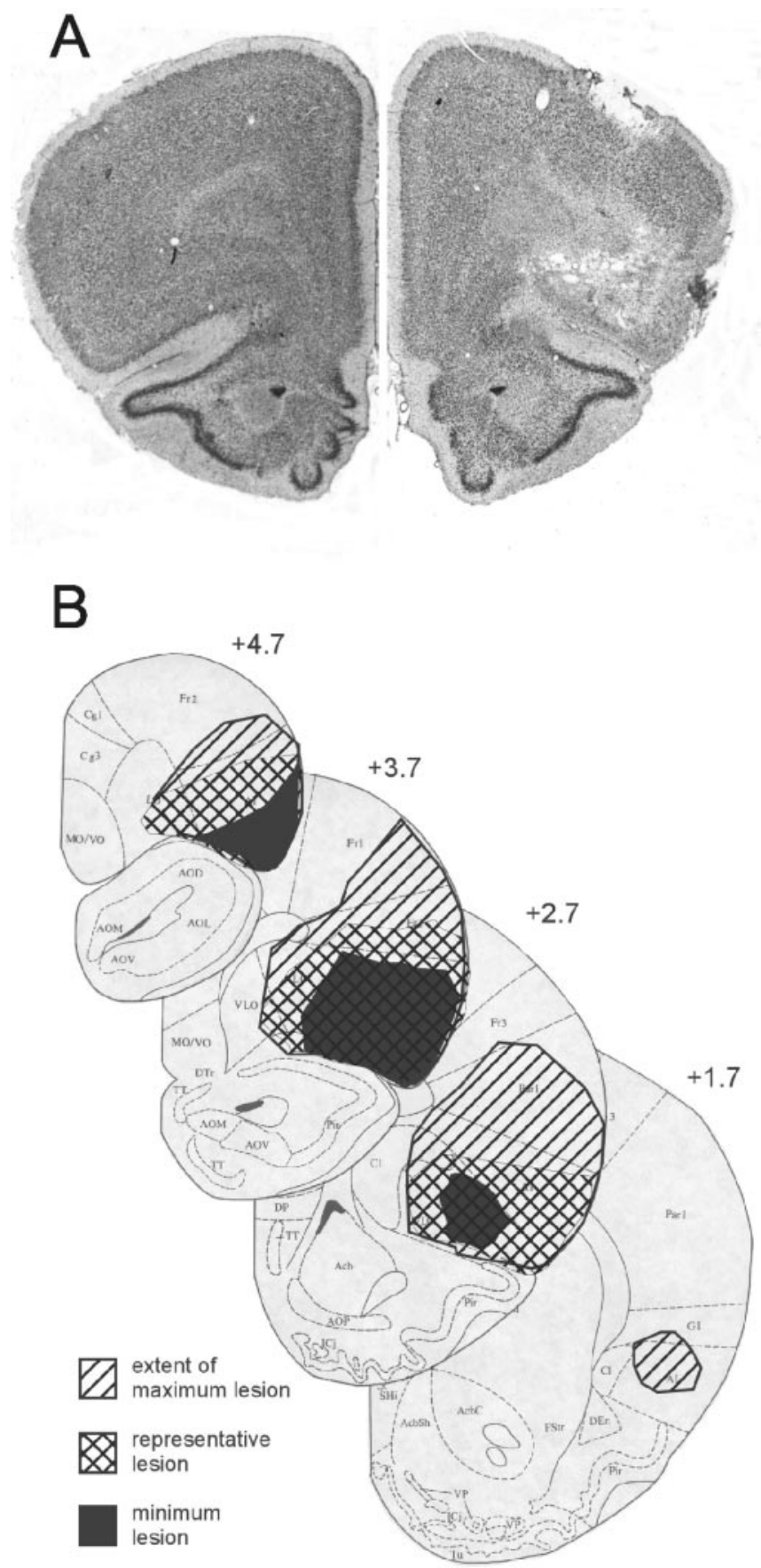

Figure 1. A, Representative neurotoxic (right hemisphere) and sham (left hemisphere) lesions of the OFC. $B$, Extents of minimum, maximum, and representative OFC lesions at various distances anterior to bregma.

$\left(F_{(1,30)}=5.74 ; p<0.05\right)$ in the sham-paired rats $(2.8 \pm 1.5 \%)$ than in the sham-unpaired rats $(17.1 \pm 6.2 \%)$, whereas pre-CS responding in the OFC-paired $(12.57 \pm 3.9 \%)$ and OFC-unpaired $(10.9 \pm 3.5 \%)$ rats did not $\operatorname{differ}(F<1)$. It is possible that these data represent an effect of devaluation on responses associated with the context in which conditioning occurred in sham, but not OFC-lesioned, rats. However, it seems more likely that those data may reflect an exaggeration of the statistically unreliable differences observed before the taste aversion learning and surgical phases (Table 2), which could only be attributable to sampling error. In support of that claim, no such differences in 
Table 2. Mean \pm SEM responding in the final conditioning session in experiments 1 and 2

\begin{tabular}{llllll}
\hline & \multicolumn{2}{l}{ OFC lesions } & & \multicolumn{2}{l}{ BLA lesions } \\
\cline { 2 - 3 } & CS & Baseline & & CS & Baseline \\
\hline Sham-paired & $78.2 \pm 2.1$ & $20.0 \pm 5.7$ & & $56.2 \pm 2.9$ & $23.5 \pm 7.3$ \\
Sham-unpaired & $77.9 \pm 5.0$ & $33.6 \pm 11.0$ & & $56.8 \pm 7.3$ & $23.6 \pm 6.3$ \\
Lesion-paired & $74.9 \pm 5.5$ & $21.1 \pm 5.6$ & & $53.6 \pm 4.2$ & $23.1 \pm 4.4$ \\
Lesion- unpaired & $73.1 \pm 5.2$ & $15.8 \pm 5.0$ & & $61.9 \pm 5.1$ & $29.9 \pm 10.1$ \\
\hline
\end{tabular}

Entries are percentage time in the food cup during the last 5 sec of the light $C S$ and in the 5 sec before each $C S$ delivery (baseline).
A

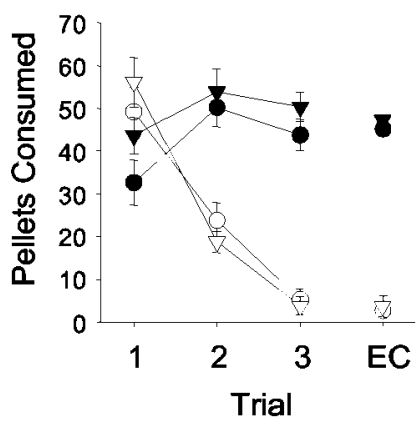

B

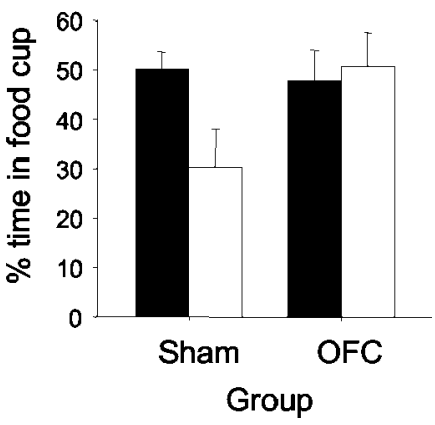

Figure 2. Data are shown for experiment 1. A, Taste aversion training in the home cage is shown on the left with the connected lines. The taste aversion test in the experimental chamber is shown with the free-floating symbols. Triangles represent sham-lesioned control groups, and circles represent the $0 \mathrm{FC}-$ lesioned groups. Black symbols represent unpaired groups, and white symbols represent paired groups. Rats in both lesion conditions reduced consumption of the food if it was paired with toxin, and both groups maintained consumption if the food was not paired with toxin. $B$, Responding to the light in the devaluation test. Black bars represent unpaired groups, and white bars represent paired groups. The control group for which food was devalued reduced conditioned responding relative to the unpaired control group. In contrast, the OFC-lesioned groups exhibited conditioned responses that did not differ as a result of the taste aversion.

pre-CS responding were observed in probe tests in experiment 2 of this study, or in the studies of Hatfield et al. (1996) or Gallagher et al. (1999). Regardless, differences in pre-CS responding do not compromise the lesion effect on devaluation of responding during the light CS; unlike pre-CS responding, there was no hint of differences in CS responding among the various groups before taste aversion and surgical treatment (Table 2).

\section{Experiment 2: effect of postconditioning BLA lesions \\ BLA lesion histology}

One of the 19 operated control rats did not survive surgery; data from the 18 remaining sham control rats (10 paired and 8 unpaired) and the 1 (unpaired) unoperated control were included in the analysis. Of the 42 BLA-lesioned rats, 1 rat did not survive surgery, and a second rat was excluded for failing to eat during the home cage taste aversion training (which made taste aversion training impossible). Twenty-four (14 paired and 10 unpaired) of the remaining lesioned rats had acceptable lesions. Lesions averaged $90 \%$ damage and included the lateral, basal, and accessory basal nuclei. Lesions were excluded $(n=16)$ if there was $<75 \%$ damage to the BLA or if there was more than minimal bilateral damage to the central nucleus of the amygdala or surrounding cortical areas. Examples of a representative lesion and sham brain are seen in photomicrographs in Figure 3, $a$ and $b$.

Presurgical performance (light-food conditioning)

Rats increased food cup responses during the light over the course of training similarly in all groups. Neither the course of acquisition nor performance on the final conditioning session (Table 2) differed as a function of later lesion status or assignment to paired or unpaired taste aversion conditions. Separate lesion $X$ taste aversion ANOVAs of responding during both the CS and pre-CS periods found no significant main effects or interactions (all $p>0.05$ ).

\section{Postsurgical performance (taste aversion and devaluation probe test)}

Taste aversion training (Fig. $4 \mathrm{~A}$ ) caused an equivalent reduction of consumption in sham and BLA-paired animals over the course of aversion training, whereas neither of the unpaired groups showed a reduction in consumption. A lesion $\times$ taste aversion $\times$ trial ANOVA found a significant effect of taste aversion $\left(F_{(1,39)}=\right.$ $60.05 ; p<0.001)$, trial $\left(F_{(2,78)}=12.10 ; p<0.001\right)$, and a significant interaction of taste aversion $\times \operatorname{trial}\left(F_{(2,78)}=76.05 ; p<\right.$ 0.001 ). No other main effects or interactions were significant (all $p>0.05)$. As in experiment 1 , the taste aversion transferred readily from the home cage in which it was established to the experimental chamber, and that transfer was unaffected by BLA lesions. A lesion $\times$ taste aversion ANOVA of the experimental chamber consumption test (Fig. $4 A$, rightmost points) found a significant effect of taste aversion $\left(F_{(1,39)}=433.86 ; p<0.001\right)$. No other main effects or interactions were significant (all $p>$ 0.05).

Figure $4 B$ shows the results of the first eight trials of the probe test session for devaluation, as in experiment 1. Unlike OFC lesions (experiment 1), which eliminated devaluation effects, BLA lesions made after light-food training had no measurable effect on devaluation. There was a significant devaluation effect in both the sham and BLA-lesioned rats. Both sham-paired $\left(F_{(1,39)}=\right.$ 5.27; $p<0.05)$ and BLA-paired $\left(F_{(1,39)}=8.62 ; p<0.01\right)$ rats showed lower levels of responding during the light CS than their respective unpaired controls. Likewise, responding of shampaired and BLA-paired rats did not differ significantly $(F<1)$. Finally, unlike in experiment 1 , there were no differences in pre-CS responding across the four groups (range, $12.4 \pm 6.4$ to $18.2 \pm 4.6 ; F<1)$.

\section{Additional control data}

In our previous assessment of the effects of pretraining BLA lesions on devaluation (Hatfield et al., 1996), taste aversion training and devaluation probe tests were conducted just 1 week after light-food conditioning. That interval was extended by 2 weeks in the current study to include surgery and postoperative recovery. To evaluate the possibility that a failure to find an effect of posttraining BLA lesions might be related to this extended interval, we tested an additional 21 BLA-lesioned and 18 sham rats. These rats received surgery before light-food conditioning, as in the study by Hatfield et al. (1996), but did not undergo taste aversion training and devaluation probe testing until 3 weeks after conditioning. Devaluation effects were eliminated in these rats, as in Hatfield et al. (1996). Consistent with that previous study, rats with BLA lesions in the devalued (paired) condition had high levels of conditioned responding during the devaluation probe tests $(39.3 \pm 8.5 \%)$ relative to the comparable shamlesioned rats $(14.4 \pm 3.9 \%)\left(F_{(1,32)}=9.79 ; p<0.01\right)$; pre-CS responding of those groups was $11.7 \pm 3.7 \%$ and $10.8 \pm 3.6 \%$, respectively $(F<1)$. Thus, the failure to observe behavioral effects of BLA lesions made after light conditioning were not attributable to the extended interval between conditioning and devaluation phases. 


\section{Discussion}

Previous work has shown that BLA and OFC lesions made before learning a lightfood relationship do not affect the acquisition of conditioned food cup responding to the light but do impair the sensitivity of that responding to reinforcer devaluation (Hatfield et al., 1996; Gallagher et al., 1999; Blundell et al., 2003). Rats with such lesions fail to exhibit the spontaneous decrease in conditioned responding to the light cue observed in controls after taste aversion training. Here, we have extended those findings by showing that when lesions are made after light-food conditioning, devaluation probe test performance is impaired by OFC lesions but not by BLA lesions. The results indicate that the OFC and BLA play different roles in mediating normal goal-directed performance in this, and likely other, settings. The BLA seems to be critical to forming representations that link cues to the incentive properties of outcomes but not for maintaining such representations in memory, updating them with new information, or for using that information to guide behavior. In contrast, the OFC seems to be essential for one or more of the latter processes, possibly in addition to being involved in the initial formation of CS-reinforcer representations.

\section{The role of the BLA and OFC in incentive learning}

The observation that performance in the devaluation task is impaired when BLA lesions are made before light-food training, but not when they are made after that training, complements the recent findings of Setlow et al. (2002). In that study, BLA lesions made before, but not after, light-food training subsequently diminished the ability of the light CS to reinforce the acquisition of second-order conditioning to a tone. These two tasks, devaluation and second-order conditioning, are similar in that both use subsequent learning and behavior to assess associative representations acquired during the initial light-food training. In both the current study and the previous study, effects of BLA lesions may have a common basis. Lesions made before light-food training prevent the CS from gaining access to motivational properties of the food reinforcer during learning, whereas lesions made after light-food training have no effect once that learning is established.

Our findings support a broad view that intact BLA function is critical in the acquisition of emotionally significant information but further suggest that its involvement in the long-term use of that information may not be critical in all settings (Cahill and McGaugh, 1998; Miserendino et al., 1990). Post-training BLA lesions have been found to disrupt display of previously learned behavior in some settings (Maren et al., 1996; Meil and See, 1997; LeDoux, 2000), and there is ample electrophysiological evidence for enduring changes in the BLA as a result of associative learning in a number of conditioning preparations (Schoenbaum et al., 1998, 1999, 2000; LeDoux, 2000). But, the lack of an effect of
B
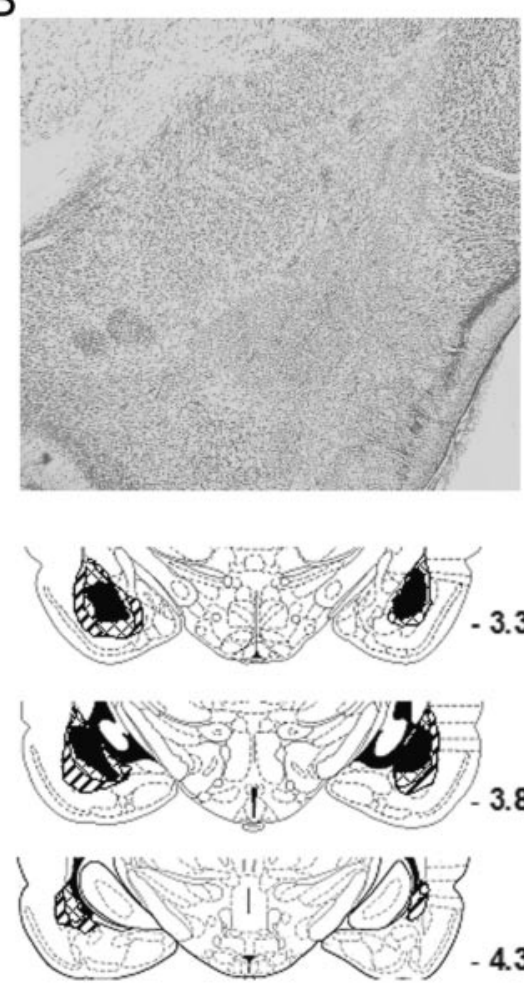

$-4.3$

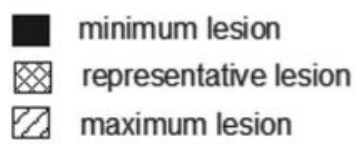

minimum lesion

DA maximum lesion

Figure 3. Representative sham $(A)$ and neurotoxic $(B)$ lesions of the BLA. $C$, Extents of minimum, maximum, and representative BLA lesions at various distances posterior to bregma.
A

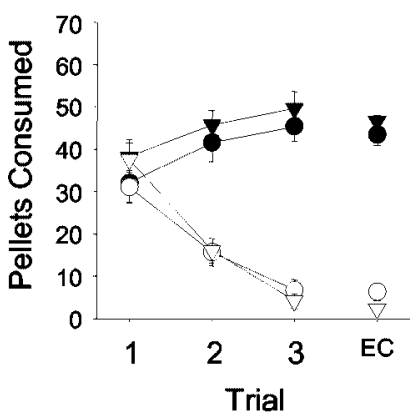

B

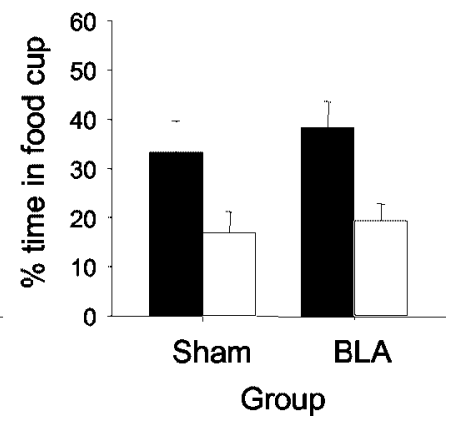

Figure 4. Data are shown for experiment 2. A, Taste aversion training in the home cage is shown on the left with the connected lines. The taste aversion test in the experimental chamber is shown with the free-floating symbols. Triangles represent sham-lesioned control groups, and circles represent the BLA-lesioned groups. Black symbols represent unpaired groups, and white symbols represent paired groups. Rats in both lesion conditions reduced consumption of the food if it was paired with toxin, and both groups maintained consumption if the food was not paired with toxin. $B$, Responding to the light in the devaluation test. Black bars represent unpaired groups, and white bars represent paired groups. Both the control and BLA-lesioned groups for which food was devalued reduced conditioned responding relative to their respective unpaired groups.

post-training BLA lesions in experiment 2 is consistent with the idea that sufficient substrates of that learning elsewhere in the brain can support behavioral functions after BLA damage, as long as the BLA is available during learning (Cahill and McGaugh, 1998). 
The OFC is one candidate region in which representations that depend on the BLA for acquisition might persist independent of the BLA to guide later performance. Consistent with this possibility, we have demonstrated previously that neurons in the BLA and OFC in rats play different roles in encoding outcomerelated information about cues in a simple go/no-go discrimination task (Schoenbaum et al., 1999, 2000). This task requires the rats to inhibit responses to odor cues that predict negative outcomes, while responding to odor cues that predict positive outcomes. During discrimination learning, BLA neurons rapidly develop cue-selective firing, whereas OFC neurons become cue selective more slowly and typically only after the behavioral criterion has been met (Schoenbaum et al., 1999). We interpreted this differential activation to reflect the rapid encoding of cue significance by the BLA and the subsequent integration of this information with information about responding in the OFC. Consistent with this idea, we recently reported the results from a study (Schoenbaum et al., 2003) in which we recorded neural activity in the OFC in the same discrimination task in rats with neurotoxic lesions of the BLA. We found that neural correlates of cue significance in the OFC were substantially disrupted in the rats with BLA lesions. Most intriguing with regard to the current report, we found that OFC representations of the outcome (i.e., neurons that fired in expectation of the outcome) failed to become associatively activated by the predictive odor cue in the BLA-lesioned rats. This result is precisely what one would expect if the BLA normally provides information about cue-outcome relationships to support the formation and maintenance of cueoutcome representations in connected brain regions such as the OFC. Moreover, these findings in electrophysiology predict that OFC lesions made after training might impair behavior when adjustments are required to reflect changes in outcome value in other tasks, such as reversal of the contingencies in the go/no-go task and, as seen in the current study, the devaluation task.

\section{The role of the OFC-BLA circuit in human behavior}

Connections between the OFC and BLA have been noted to be important for emotional or affective learning in primate species (Baxter et al., 2000; Ghashghaei and Barbas, 2002). Our observation of the separate roles of the BLA and OFC in the devaluation task may have useful implications for understanding the involvement of this circuit in complex human behavior. For example, Bechara et al. (1999) found deficits in the performance of patients with damage to the amygdala or vmPFC in a gambling task. In this task, participants pick cards from one of four decks, in which two decks provide high initial rewards but larger penalties, causing a net loss over the long run, whereas the other two decks provide lower rewards but even smaller penalties, with a net gain over the long run. Normal participants started choosing from the high reward/high penalty decks but then shifted to the low reward/low penalty decks as the advantageous strategy became obvious. Patients with amygdala or vmPFC damage also began by picking from the high reward/high penalty decks but did not shift their responding to the low reward/low penalty decks. Whereas the amygdala patients' failure to shift from an increasingly disadvantageous strategy may be related to an insensitivity to the negative consequences of their behavior, in vmPFC patients this failure occurred despite an ability to verbalize the task contingencies and to recognize the negative consequences of their behavior. This pattern of deficits is compatible with the view that the BLA is necessary for the initial encoding of the emotional consequences of events or actions and that the OFC is critical for adaptive change in behavior as those consequences are experienced. Be- chara et al. (1999) argued that such inability to adjust behavior according to its consequences may underlie deficits in social behavior and poor judgment often found in patients with vmPFC (Rolls et al., 1994) or amygdala (Tranel and Hyman, 1990) damage. A better understanding of the interaction of these brain regions in a model system like that used here may elucidate the specific basis for these impairments and might inform the development of methods to improve the social abilities and decision making of such individuals.

\section{References}

Baxter MG, Parker A, Lindner CC, Izquierdo AD, Murray EA (2000) Control of response selection by reinforcer value requires interaction of amygdala and orbital frontal cortex. J Neurosci 20:4311-4319.

Bechara A, Damasio H, Damasio AR, Lee GP (1999) Different contributions of the human amygdala and ventromedial prefrontal cortex to decisionmaking. J Neurosci 19:5473-5481.

Blundell P, Hall G, Killcross S (2003) Preserved sensitivity to outcome value after lesions of the basolateral amygdala. J Neurosci 23:7702-7709.

Cahill L, McGaugh JL (1998) Mechanisms of emotional arousal and lasting declarative memory. Trends Neurosci 21:294-299.

Gallagher M, McMahan RW, Schoenbaum G (1999) Orbitofrontal cortex and representation of incentive value in associative learning. J Neurosci 19:6610-6614.

Ghashghaei HT, Barbas H (2002) Pathways for emotion: interactions of prefrontal and anterior temporal pathways in the amygdala of the rhesus monkey. Neuroscience 115:1261-1279.

Hatfield T, Han J-S, Conley M, Gallagher M, Holland P (1996) Neurotoxic lesion of the basolateral, but not central, amygdala interfere with Pavlovian second-order conditioning and reinforcer-devaluation effects. J Neurosci 16:5256-5265.

Holland PC (1977) The conditioned stimulus as a determinant of the form of the Pavlovian conditioned response. J Exp Psychol Anim Behav Process 3:77-104.

Holland PC, Straub JJ (1979) Differential effects of two ways of devaluing the unconditioned stimulus after Pavlovian appetitive conditioning. J Exp Psychol Anim Behav Process 5:65-78.

Jones B, Mishkin M (1972) Limbic lesions and the problem of stimulusreinforcement associations. Exp Neurol 36:362-377.

Kita H, Kitai ST (1990) Amygdaloid projections to the frontal cortex and the striatum in the rat. J Comp Neurol 298:40-49.

Kosar E, Grill HJ, Norgren R (1986a) Gustatory cortex in the rat. I. Physiological properties and cytoarchitecture. Brain Res 379:329-341.

Kosar E, Grill HJ, Norgren R (1986b) Gustatory cortex in the rat. II. Thalmocortical projections. Brain Res 379:342-352.

Krettek JE, Price JL (1977) Projections from the amygdaloid complex to the cerebral cortex and thalamus in the rat and cat. J Comp Neurol 172:225-254.

Krushel LA, Van Der Kooy D (1988) Visceral cortex: integration of the mucosal senses with limbic information in the rat agranular insular cortex. J Comp Neurol 270:39-54.

LeDoux JE (2000) The amygdala and emotion: a view through fear. In: The amygdala: a functional analysis (Aggleton JP, ed), pp 289-310. New York: Oxford UP.

Malkova L, Gaffan D, Murray E (1997) Excitotoxic lesions of the amygdala fail to produce impairment in visual learning for auditory secondary reinforcement but interfere with reinforcer devaluation effects in rhesus monkeys. J Neurosci 17:6011-6020.

Maren S, Aharonov G, Stote DL, Fanselow MS (1996) N-methyl-Daspartate receptors in the basolateral amygdala are required for both acquisition and expression of conditional fear in rats. Behav Neurosci 110:1365-1374.

Meil WM, See RE (1997) Lesions of the basolateral amygdala abolish the ability of drug-associated cues to reinstate responding during withdrawal from self-administered cocaine. Behav Brain Res 87:139-148.

Miserendino MJ, Sananes CB, Melia R, Davis M (1990) Blocking of acquisition but not expression of conditioned fear-potentiated startle by NMDA antagonists in the amygdala. Nature 345:716-718.

Rolls ET, Hornak J, Wade D, McGrath J (1994) Emotion-related learning in patients with social and emotional changes associated with frontal lobe damage. J Neurol Neurosurg Psychiatry 57:1518-1524. 
Saper CB (1982) Convergence of autonomic and limbic connections in the insular cortex of the rat. J Comp Neurol 210:163-173.

Schoenbaum G, Chiba AA, Gallagher M (1998) Orbitofrontal cortex and basolateral amygdala encode expected outcomes during learning. Nat Neurosci 1:155-159.

Schoenbaum G, Chiba AA, Gallagher M (1999) Neural encoding in orbitofrontal cortex and basolateral amygdala during olfactory discrimination learning. J Neurosci 19:1876-1884.

Schoenbaum G, Chiba AA, Gallagher M (2000) Changes in functional connectivity in orbitofrontal cortex and basolateral amygdala during learning and reversal training. J Neurosci 20:5179-5189.

Schoenbaum G, Setlow B, Saddoris MP, Gallagher M (2003) Encoding pre- dicted outcome and acquired value in orbitofrontal cortex during cue sampling depends upon input from basolateral amygdala. Neuron, 39:855-867.

Setlow B, Gallagher M, Holland PC (2002) The basolateral complex of the amygdala is necessary for acquisition but not expression of CS motivational value in appetitive Pavlovian second-order conditioning. Eur J Neurosci 15:1841-1853.

Shi CJ, Cassell MD (1998) Cortical, thalamic, and amygdaloid connections of the anterior and posterior insular cortices. J Comp Neurol 399:440-468.

Tranel D, Hyman BT (1990) Neuropsychological correlates of bilateral amygdala damage. Arch Neurol 47:349-355. 